\title{
Regulation associated modules reflect 3D genome modularity associated with chromatin activity
}

\author{
Lina Zheng ${ }^{1}$, Wei Wang ${ }^{1,2,3,{ }^{*}}$
}

${ }^{1}$ Bioinformatics and Systems Biology program, University of California, San Diego, La Jolla, CA 92093-0359.

2 Department of Chemistry and Biochemistry, University of California, San Diego, La Jolla, CA 92093-0359.

${ }^{3}$ Department of Cellular and Molecular Medicine, University of California, San Diego, La Jolla, CA 92093-0359.

*Correspondence: wei-wang@ucsd.edu 


\section{ABSTRACT}

The 3D genome has been shown to be organized into modules including topologically associating domains (TADs) and compartments that are primarily defined by spatial contacts from $\mathrm{Hi}-\mathrm{C}$ or other experiments. There exists a gap to investigate whether and how the spatial modularity of the chromatin is related to the functional modularity resulting from the chromatin activity. Increasing evidence shows a tight interplay between histone modifications and $3 \mathrm{D}$ chromatin organization. As the histone modifications reflect the chromatin activity, it is tempting to infer the spatial modularity of the genome directly from the histone modification patterns, which would establish the connection between the spatial and functional modularity of the genome. However, uncovering the 3D genomic modules using histone modifications has not been well explored. Here, we report that the histone modifications show a modular pattern (referred to as regulation associated modules, RAMs) that reflects the spatial modularity of the chromatin structure. We found that enhancer-promoter interactions and extrachromosomal DNAs (ecDNAs) occur more often within the same RAMs than within the same TADs, indicating stronger insulation of the RAM boundaries and a modularization of the $3 \mathrm{D}$ genome at a scale better aligned with the chromatin activity. Consistently, compared to the TAD boundaries, in silico predictions showed that deletions of RAM boundaries perturb the chromatin structure more severely and somatic variants in the cancer samples are more enriched in the RAM boundaries. These observations suggest that RAMs reflect a modular organization of the $3 \mathrm{D}$ genome at a scale better aligned with chromatin activity, providing a bridge connecting the structural and functional modularity of the genome. 


\section{INTRODUCTION}

Histone modifications are critical to shape the chromatin structure and regulate gene expression(1, 2). Active marks such as H3K27ac and H3K4me3 open up chromatin to allow access of transcription factors (TFs) and transcription machinery to promoters or enhancers. Repressive marks such as $\mathrm{H} 3 \mathrm{~K} 9 \mathrm{me} 3$ and $\mathrm{H} 3 \mathrm{~K} 27 \mathrm{me} 3$ condensate chromatin to block TF binding and suppress gene expression. DNA marked by active and repressive histone modifications form euchromatin and heterochromatin that are distinct on the compactness. These observations suggest that histone modifications have an important impact on organizing the regional and global 3D genome.

Accumulating evidence has revealed the association of histone modifications with the topologically associating domains (TADs)(3-6) and compartments $(7,8)$ derived from the $\mathrm{Hi}-\mathrm{C}$ contact maps showing plaid patterns. TADs represent genomic domains forming dense internal contacts but fewer contacts with neighboring regions. The TAD boundaries are demarcated with CTCF sites or active transcribed DNA sequences. The Hi-C data also shows that the $3 \mathrm{D}$ genome is partitioned into transcriptionally active (compartment A) and suppressed (compartment B) compartments. Active and repressive histone marks are enriched, but do not exclusively appear, in the A and B compartments, respectively $(7,8)$. Computational models have shown that histone modification signals are predictive of $\mathrm{Hi}-\mathrm{C}$ contacts particularly for enhancer-promoter interactions(9), TAD boundaries(10) and compartments(11). Histone modifications are tightly associated with transcriptional activity (12-14) while transcription and proteins involved in transcriptional regulation including RNA polymerase and TFs have been shown to contribute to compartmentation and active promoters and enhancers tend to form clusters in the nucleus(15-18).

Despite the mechanisms underlying the interplay between histone modifications and chromatin organization remain elusive, histone modifications can indicate the spatial organization of the genome as readout signals for regulatory modules. However, the current analysis has been limited to associating histone marks to $\mathrm{Hi}-\mathrm{C}$ derived TADs and compartments. An unfilled gap is to use histone modifications to directly elucidate the modular organization of the $3 \mathrm{D}$ genome. We propose here to define the spatial module 
of the genome organization resulting from the chromatin activities reflected by histone modifications.

We found that the frequency profiles of the H3K27ac peaks present a modular structure (referred to as regulation associated modules, RAMs). A large number of these modules are shared across cell types and can be independently derived using other active histone marks, including H3K4me3 and H3K4me1. We uncovered several lines of evidence to support the hypothesis that the RAMs are spatial modules resulting from functional activities: the enhancer-promoter interactions dominantly occur within RAMs; the extrachromosomal DNAs (ecDNAs) tend to be originated from the same RAMs rather than split in multiple RAMs; RAMs are resistant to cohesin degradation. These properties of RAMs distinguish them from TADs and compartments. Furthermore, deletion of the RAM boundaries is predicted to alter the chromatin organization more significantly than the deletion of TAD boundaries. Consistently, the somatic genetic variations in cancer patients are enriched in RAM boundaries, suggesting a possible mechanism of tumorigenesis involved in altering the chromatin modules.

\section{RESULTS}

\section{Regulation associated modules (RAMs) detected by the histone modification peaks}

We analyzed the density profile of H3K27ac peaks (i.e. peak count in a sliding window) from chromatin immunoprecipitation assays with sequencing (ChIP-Seq) experiments as using the peak density instead of read count density can better remove the noise from the background signals. We downloaded ChIP-seq data of 93 normal and 19 cancer samples from Roadmap Epigenomics Project (http://www.roadmapepigenomics.org/)(19) and ENCODE portal (https://www.encodeproject.org/)(20) (Table S1). Using a sliding window (a fixed flanking size of $500 \mathrm{kbp}$ and step size varying from $10 \mathrm{kbp}$ to $500 \mathrm{kbp}$ ), we computed the H3K27ac peak densities in the linear genome. Regardless of the step size, the H3K27ac peak densities were not evenly distributed and showed a modular pattern (Figure 1A). The active marks of H3K4me1, H3K4me3 and H3K36me3 showed similar peak density profiles to H3K27ac in the 93 samples, indicated by high Pearson correlations between them, whereas the repressive marks of $\mathrm{H} 3 \mathrm{~K} 27 \mathrm{me} 3$ and $\mathrm{H} 3 \mathrm{~K} 9 \mathrm{me} 3$ 
had less consistent patterns (Figure 1B). Given the highly correlated active mark patterns, we focused on analyzing the H3K27ac signals as the other active marks show similar modular structure.

At a given step size, we identified the valley or minima of the H3K27ac peak profile that was smoothed using local polynomial fit in each chromosome and in each cell type (see Materials and Methods). These valleys demarcated the boundaries of the modular domains (called Regulation Associated Domains or RAMs). We varied the step size from $10 \mathrm{kbp}$ to $500 \mathrm{kbp}$ and fixed the window size to $500 \mathrm{kbp}$. It is not surprising that, with the increasing step size, the RAM size increased and a higher percentage of RAMs were shared between samples (fig S1-S2). We observed that the number of common RAMs in all the chromosomes reached a plateau at $250 \mathrm{kbp}$ step size in both normal and cancer samples, which indicates the identified RAMs are most conserved across diverse cell types (fig S3A-B). We thus used this step size of $250 \mathrm{kbp}$ for the remaining analyses. A RAM boundary is called consensus RAM (CRAM) boundary if it is shared by $>25 \%$ of the samples. This way, 711 cRAMs were detected in the normal samples and 771 cRAMs in the cancer samples (see Materials and Methods). On average, 60\% of the RAMs in a cell type are consensus (referred to as cRAMs) and the remaining cell-type specific (fig S3C).

One example of the identified RAMs in chr12 of the GM12878 cell by IGV software(21) is shown in Figure 1C-E. Obviously, the RAM boundaries have lower signals of the active histone marks (H3K27ac, H3K4me1, H3K4me3, H3K36me3) and higher repressive marks (H3K9me3 and H3K27me3) compared to the within RAM regions. Consistently, they tend to align with the B compartment or subcompartments (B1, B2, B3). Furthermore, by counting the number of the 3D contacting neighbors for each locus using the $10 \mathrm{~kb}$ resolution Hi-C data in GM12878 (contacts with $\log (P$-value) <=-10), we found that the RAM boundaries tend to harbor many 3D contacts, indicated an enrichment with densely packed DNA sequences forming many spatial contacts (Figure 1C).

\section{Characterization of the consensus regulation associated modules (cRAMs)}


As cRAMs are largely shared between diverse cell lines, we further characterized them. Among the cell lines that have both active and repressive marks (all are normal cells), as expected, we found that the cRAM boundaries have lower peak density of the active marks (H3K27ac, H3K4me3, H3K4me1 and H3K36me3) and slightly higher peak densities of repressive marks (H3K27me3 and H3K9me3) than the cRAM regions (see examples in GM12878 and HUVEC cell lines in Figure 2A). To quantify the difference of the histone modifications among the cRAM boundaries and the non-boundary regions, we counted the peak density using a sliding window, and compared the histone modifications enrichment across 93 normal samples. The P-value $<0.05$ from the Wilcoxon Rank Sum test indicated that cRAM boundaries have significantly lower active marks and higher repressive marks than the non-boundaries of cRAMs (Figure 2B-G). Furthermore, using the available 10kb-resolution $\mathrm{Hi}-\mathrm{C}$ data in the K562, GM12878, A549, IMR90, NHEK, HUVEC, HMEC and HCT116 cell lines, we found that the cRAM boundaries have significantly more Hi-C contacts (intrachromosomal contacts with log(P. value)<=-10) compared to the whole genome (Figure $\mathbf{2 H}$ ), which is consistent with the genome browser view for any RAM boundary in Figure 1C. These observations suggested that the cRAM boundaries are formed by densely packed DNA sequences harboring many 3D contacts.

We next investigated how RAMs are related to the previously identified chromatin modules. First, the median size of cRAMs ( $3.3 \mathrm{Mbp})$ is larger than TADs ( 600kbp) and one RAM often spans across multiple TADs (Figure 2I). Second, using the Hi-C data, we identified the A/B compartments at $250 \mathrm{~kb}$ resolution (see Materials and Methods). We calculated the percentage of the A and B compartments in each cRAM (250kb bin size) across the cell types. While the A-compartments account around $50 \%-75 \%$ in each of the cRAM, a single cRAM is largely composed by a mixture of A and B compartments, indicating a distinction between cRAMs and compartments (Figure 2J). Consistently, the cRAM boundaries are enriched with B-compartment but also with a significant portion of A compartments (Figure 2J). Third, we checked the Lamin-B1 signals for the cRAM boundaries. Lamin-B1 is a scaffolding component of the nuclear envelope(22, 23). A positive signal for Lamin-B1 suggests a close distance to the nuclear lamina, which could 
be used to define lamina associated domain (LAD). When aligning the CRAM boundaries with the Lamin-B1 signals (see Materials and Methods), we found on average 69\% of the cRAM boundaries overlapping with Lamin-B1 signals across the cell types and meanwhile on average $62.7 \%$ of the LADs identified from each cell type overlapping with cRAM boundaries (Figure 2K), indicating that LADs and cRAMs are also different. Taken together, the cRAM boundaries are formed by densely packed DNA sequences; while they are enriched with B compartment and Lamin-B1 signals, RAMs are clearly distinct from the previously reported domain structures such as TADs, LADs and A/B compartments.

\section{RAMs are functional units}

If RAMs are functional modules, we reason that the majority of the promoter-enhancer interactions should occur within the same RAMs. We downloaded 970 high-confidence promoter-enhancer interactions in the K562 cell line that were experimentally validated in ref $(24,25)$ and $95 \%$ of them are located within the same RAMs, compared to $75 \%$ of them in the same $\operatorname{TADs}(4,6,8,26)$ (Figure 3A-B). Two examples of promoter-enhancer interactions are shown in Figure 3G: the enhancer-promoter interactions of STEAP1B and VGF are across multiple TADs but within the same K562 RAMs marked by continuous strong H3K27ac peaks. This observation suggests that RAMs may represent regulatory modules and RAM boundaries insulate promoter-enhancer contacts across RAMs at a scale more appropriate than TADs to capture functional modularity of chromatin activity.

We further investigated how the modularity defined by RAMs affects gene expression. To this end, we examined whether the enhancer-promoter pairs located within the same K562 RAMs but separated by RAM boundaries in other cells would specifically impact gene expression in K562. When comparing K562 to the normal cell line GM12878, we found 885 K562 enhancer-promoter interactions were within the same RAMs in both cell lines and 39 only in K562 (Figure 3C). The majority of the genes regulated by the 39 enhancer-promoter interactions are upregulated in K562 compared to in GM12878 (Pvalue $=0.0002$ by Hypergeometric Test, see Materials and Methods) (Figure 3D), 
indicating that the RAM organization facilitates promoter-enhancer interactions to activate gene expression. For example, the RAB31 promoter interacts with an enhancer that is located within the same RAM in K562 but in a RAM boundary in GM12878 where the enhancer would be silenced in GM12878; the PRELID2 promoter and its interacting enhancer are located within the same K562 RAM but reside in a GM12878 RAM boundary indicating suppression in GM12878 (Figure $3 \mathrm{H}$ ). In fact, the RAB31 and PRELID2 normalized expression levels are 34.29 and 5.37 folds higher in K562 than in GM12878, respectively. All the upregulated gene expressions involved in enhancer-promoter interactions that occurred in the same RAM in K562 but in different GM12878 RAMs are shown in Table S2. We had a similar observation by comparing K562 and HEPG2: while the majority of the promoter-enhancer pairs are intra-RAM in both cell lines, 28 are only occurred in the same RAM in K562 but in different HEPG2 RAMs (Figure 3E) and the corresponding genes have higher expressions in K562 than in HEPG2 (P-value $=0.0006$ by Hypergeometric Test) (Figure 3F, Table S3). Furthermore, we examined the K562 enhancer-promoter pairs in K562-specific RAMs and the cancer consensus RAMs (cancer cRAMs). 750 pairs were identified as intra-RAM interactions in both K562 RAMs and cancer cRAMs (fig S4A). 77 genes were involved in the 174 pairs that are only intraRAM interactions in K562, and 20 out of the 77 genes were detected as K562 specifically highly expressed genes across 92 cancer cell lines (hypergeometric test $P$-value $=0.03$ ) documented in the Harmonizome database(27) (fig S4B). These observations further illustrated that RAMs represent a modularity directly associated with functional activity of the chromatin.

\section{RAMs are insensitive to cohesin degradation}

Previous studies showed that cohesin degradation would disrupt loop domains and topological associated domains (TADs) but largely not change histone modifications and gene expression $(28,29)$. Therefore, RAMs are not expected to be affected by cohesin degradation. For confirmation, we identified RAMs using H3K27ac data in the HCT-116 RAD21-mAC cells untreated and treated for 6 hours with IAA. The RAM patterns for each chromosome were highly correlated between treated and untreated cells (fig S5A) and the recall rate for the RAM boundaries was 0.9 on average for all the chromosomes (fig 
S5B). This observation indicates that the RAM formation is independent from cohesin, distinguishing RAMs from TADs and loop domains.

\section{Extrachromosomal DNA (ecDNA) from cancer patients majorly originated from intra-RAM}

Circular extrachromosomal DNAs (ecDNAs) are prevalent in tumors and their length ranges from $100 \mathrm{kbp}$ to megabases, and the genes encoded in the ecDNAs are often amplified in cancers(30-32). We reason that, if RAMs are functional modules, ecDNAs would form within RAMs because RAM boundaries are highly condensed DNAs that would restrain the transcription of genes residing in ecDNAs. To test this hypothesis, we downloaded the ecDNAs identified from cancer patients(33), and filtered the ecDNAs corresponding to the median size of the cancer cRAM length (2.5Mb), i.e. only ecDNAs with size $<2.5 \mathrm{Mb}$ were kept (i.e. $78 \%$ of all the ecDNAs). We found that $98 \%$ of 2459 ecDNAs were located within the individual RAMs. As a comparison, we performed the same analysis on TADs. We took the conserved TADs defined in the Dixon et al. study(4) and only kept the ecDNAs shorter than $880 \mathrm{kbp}$ (68\% of all the ecDNAs), the median size of the TAD length. We found that $86 \%$ of the 2150 ecDNAs were within individual TADs (Figure 3J, 3K). Because the ecDNAs were filtered to have comparable length with the RAM and TAD sizes, respectively, this lower intra-domain percentage for TAD compared to RAM is not due to the larger size of RAMs. Furthermore, GREAT analysis(34) (http://great.stanford.edu/public/html/) on the ecDNAs that fall into intra-cancer cRAMs but split in TADs revealed that they are highly enriched in "positive regulation of DNA replication" with a P-value of 5.1641E-19. There are 12 genes involved in this pathway: ATF1, BMP5, BMP6, EGFR, FGFR1, GLI2, IGF1, IL6, JUN, KITLG, PDGFA, PDGFRA, which are known important for cell proliferation and cancer pathogenesis. For example, EGFR is a driver of tumorigenesis(35). Deregulation of the oncogenic FGFR signaling has been frequently observed in multiple types of cancers(36). The PDGF mediated signaling has been reported to be involved in the cell proliferation and invasion(37). The observations that ecDNAs tend to originate from intra-RAMs, which suggests that RAM is a functional module. 


\section{Deletion of the CRAM boundaries are predicted to alter the 3D chromatin structures}

To systematically examine the impact of deleting cRAM boundaries to the chromatin structure, we resorted to computational predictions using a deep learning model ORCA(38) (https://github.com/jzhoulab/orca) as it is prohibitive to perform hundreds of $\mathrm{Hi}-\mathrm{C}$ experiments with sufficient resolution. We took the ORCA model pre-trained on the high resolution Hi-C and Micro-C data in H1-hESC and HFF cell lines to predict 3D chromatin architecture from kilobase to whole-chromosome scale using DNA sequences. It also provided perturbation predictions if certain sequences were targeted. CRAM boundaries shared between cancer and normal samples are apparently important, therefore we selected all 418 of them that are located at least $16 \mathrm{Mb}$ away from centromere to predict their impacts on $\mathrm{Hi}-\mathrm{C}$ contacts if deleted. As a comparison, we also included $298 \mathrm{H} 1$-hESC and $187 \mathrm{HFF}$ TAD boundaries (length of the TAD boundaries >= $100 \mathrm{kbp}$; nonoverlap with selected cRAM boundaries; away from centromere at least $16 \mathrm{Mb})$ in the computational perturbations screening. Considering that the cRAM boundaries are often larger than the TAD boundaries, we only deleted the center 100kbp of CRAM and TAD boundaries to avoid bias introduced by deletion size.

To measure the similarity between the deletion and wildtype $\mathrm{Hi}-\mathrm{C}$ contact matrices, we calculated the Pearson correlation between them. Compared to the TAD deletions, deleting CRAM boundaries obviously resulted in lower correlation coefficients, indicating larger chromatin alterations, at the highest resolutions the ORCA model could predict (4kb and $8 \mathrm{~kb}$ resolutions with Wilcoxon Rank Sum test P-values of 2.4E-11 and 2.6E-7, respectively) (Figure 4B). Deletion of the cRAM boundary (chr10:115,940,000116,040,000, in hg38) on Hi-C contacts in HFF and H1-hESC cells is shown as an example (Figure 4A, fig S6). The 3D contacts are severely weakened by deleting the cRAM boundary in both cell types.

\section{Somatic genetic variations enriched in regulation associated modules boundaries}

If RAMs are functional modules important for regulating functional activities, we reason that somatic mutations in cancers may target the RAM boundaries to disrupt the modular organization of chromatin leading to aberrant regulation of gene expression and resulting 
tumorigenesis. The PCAWG study revealed consensus mutations and variations from thousands of cancer patients including 20 millions of somatic single nucleotide variations (SNVs) and $\sim 1.08$ millions of indels (https://dcc.icgc.org/releases)(39). We found that, while cancer cRAM boundaries cover $\sim 21.6 \%$ of the genome, they host $25.9 \%$ of somatic SNVs and $23.4 \%$ somatic indels. As a comparison, the conserved TAD boundaries(4) covering $6.5 \%$ genome containing $5.2 \%$ somatic SNVs and $6 \%$ somatic indels (Figure 4C). The cancer CRAM boundaries are significantly enriched with both somatic SNVs and indels compared to the TAD boundaries (P-value<1E-5 from Two-sample Proportion tests, see Materials and Methods), indicating a stronger association with cancer mutations.

To elucidate the sequence features associated with cRAM boundaries and investigate how the somatic mutations change such features, we performed motif analysis on the cRAM boundaries using Homer(40). We focused on the cRAMs that are common in cancer (normal) but not in normal (cancer) samples as cancer (normal)-specific cRAMs, as they represent changed modularity between cancer and normal samples. By comparing cancer-specific and normal-specific cRAMs, we found 73 and 74 motifs enriched only in cancer and normal specific cRAM boundaries, respectively (example motifs shown in Figure 4D, 4F). We employed FIMO(41) to identify the occurrences of the enriched motifs that counted for $25.8 \%$ and $30.1 \%$ in base pairs, respectively, in the cancer and normal specific cRAM boundaries (Figure 4E, 4G). We next mapped the PCAWG somatic SNVs and indels onto the cancer and normal specific cRAM boundaries. While somatic SNVs do not show a preferred occurrences within the enriched motifs (24.9\% and $29.9 \%$ for cancer and normal specific cRAM boundaries, respectively), the somatic indels overlapping with the cancer/normal-specific cRAM boundaries preferentially hit the enriched motifs $(52.3 \%$ and $63 \%$ for cancer and normal specific cRAM boundaries, respectively), more than two-fold by chance, in the altered cRAM boundaries between normal and cancer samples. We speculate that the enriched motifs in the cancer and normal-specific cRAM boundaries may respectively facilitate disruption and formation of cRAM boundaries in the normal samples. Two examples of these motifs overlapping with indels are shown in Figure $4 \mathrm{H}-\mathrm{I}$. We identified genes within $2.5 \mathrm{~kb}$ from the enriched motifs overlapping with somatic indels and analyzed the enriched pathways 
using g:Profiler(42). The KEGG over-represented pathways are shown in Figure 4J, and the gene ontology molecular functions are shown in fig S7. Furthermore, we downloaded the normalized gene expressions of the TCGA and GTEx samples from Expression Atlas (https://www.ebi.ac.uk/gxa/home) and identified differentially expressed genes (DEGs) ( $P$-value<=0.05 by the Wilcoxon Rank Sum tests between cancer and normal samples). The top ranked pathways associated with cancer and cell proliferation are highly enriched with the DEGs, such as PIK3AP1, LABM3, AKT1, MYB in the "PI3K-AKT pathway" (32\%) and "pathways in cancer" (20\%) (Figure 4K). These observations suggested that the motifs specifically enriched in the formation of cancer cRAM boundaries and disruption of normal cRAM boundaries are close to genes important for tumorigenesis, cell survival and cell proliferation. Somatic indels can severely alter these motifs and may contribute to the cRAM boundary change, affecting the expressions of the nearby genes.

\section{DISCUSSION}

In this study, we analyzed the peak density profiles of histone modifications data and found they show modular patterns. These modules are clearly defined by active marks such as H3K27ac, H3K4me1 and H3K4me3, indicating their association with functional activity of the genome, and are thus termed regulation associated modules (RAMs). While TADs and compartments are identified from the 3D contacts measured by Hi-C, RAMs are delineated by histone modifications that are directly related to chromatin accessibility and gene expression. We showed that RAMs are obviously distinct from TADs, compartments and LADs although some RAM boundaries do overlap with TAD, compartment or LAD boundaries.

By surveying 93 normal and 19 cancer samples, we found the following evidence to support that RAMs are spatial modules resulting from functional activities. First, we observed that on average $60 \%$ of the RAMs (i.e. consensus RAMs) are largely shared across samples, while some of them are sample specific. Compared to TADs, consensus RAMs host higher percent of experimentally confirmed promoter-enhancer contacts (i.e. within the same RAMs), suggesting RAMs represent a modularization of the genome at a scale better aligned with transcriptional regulation. Second, ecDNAs detected from cancer patients tend to originate from the same RAMs rather than across multiple RAMs, 
supporting the insulation effect of RAM boundaries. Third, deletion of the cRAM boundaries would result in more severe chromatin alteration than the TAD boundaries based on in silico predictions of $\mathrm{Hi}-\mathrm{C}$ contacts, suggesting the importance of cRAM boundaries in maintaining the chromatin structure. Fourth, cRAM boundaries are also more enriched with somatic genetic variants of SNVs and indels than the TAD boundaries. In particular, the somatic indels tend to disrupt the motifs specifically enriched in cancer or normal specific cRAM boundaries, suggesting a possible mechanism of tumorigenesis involved in altering the chromatin modularity.

To investigate the mechanisms underlying the RAM formation, we found that the RAMs are separated by densely packed DNA regions (as shown by their large number of Hi-C contacts) enriched with repressive histone modifications and lacking open chromatin, active histone marks or transcriptional events. Furthermore, unlike TADs, RAMs are insensitive to cohesin degradation. Taken together, these observations clearly show that RAMs are distinct from loop domains and TADs. RAMs are also different from lamina associated domains (LADs) defined by measuring the intermediate filament protein LMNB1 localization. The LADs are formed through interactions between chromatin and lamina, and they are located at the periphery of the genome. The RAM boundaries are demarcated by densely packed DNAs and many RAM boundaries are not located in regions interacting with lamina or overlapping with TAD boundaries, and thus the mechanism underlying these RAM boundary formation should be different from other chromatin modules including TADs, LADs and compartments.

Many studies (such as in ref $(43,44)$ ) have shown that multivalent cations such as calcium, magnesium, and manganese can reduce the electrostatic repulsions between the DNA chains and induce DNA condensation. Furthermore, these cations may bind to specific DNA sequences(45) and affect nucleosome positioning(46, 47). Therefore, a possible mechanism can be that genomic DNAs become densely packed around cations such as $\mathrm{Ca}^{2+}, \mathrm{Mg}^{2+}$ and $\mathrm{Mn}^{2+}$ to form $\mathrm{RAM}$ boundaries even if they are not marked by H3K9me3 or interacting with lamina. Proteins such as calcium binding proteins that carry many cations or their interacting partners may recognize specific DNA sequences such as those motifs enriched in the cancer or normal specific cRAM boundaries to facilitate 
locus-specific localization of cations. Interestingly, the most enriched molecular function of the genes close to $(<2.5 \mathrm{kbp})$ the enriched motifs overlapping with somatic indels in the cancer or normal specific cRAM boundaries is calcium ion binding (fig S7), and $\sim 30 \%$ of them are differentially expressed in cancer and normal samples (Supplementary Table S4), implying a possible feedback mechanism. This hypothesis and the mechanistic details are awaiting for future studies.

Acknowledgements: We are grateful to the labs of Drs. Bas van Steensel, Erez Lieberman Aiden and Marion Cremer for data sharing. Funding: This project was partially supported by NIH (R01HG009626 to W.W.). Author Contributions: W.W. conceived and supervised the project. L.Z. performed the bioinformatics analysis. L.Z. and W.W. wrote the manuscripts. Competing interests: The authors declare no competing interests. Data availability: All data needed to evaluate the conclusions in the paper are present in the paper and/or the Supplementary Materials. 


\section{Materials and Methods.}

\section{Regulatory associated modules (RAM) identification}

Data Source. The 93 normal and 19 cancer samples with the called H3K27ac narrow peaks in hg19 were downloaded from Roadmap Epigenomics portal (https://egg2.wustl.edu/roadmap/web portal/)(19) and ENCODE portal (https://www.encodeproject.org/). Table S1 lists the samples used in this study.

RAM identification in individual samples. We calculated the H3K27ac narrow-peak density using a sliding window of step size equal to $10 \mathrm{~kb}, 50 \mathrm{~kb}, 100 \mathrm{~kb}, 250 \mathrm{~kb}$ and $500 \mathrm{~kb}$ respectively and $500 \mathrm{~kb}$ flanking size of each window in every sample. The H3K27ac narrow-peak profiles were then smoothed by a local polynomial regression fitting(48). The RAM boundaries (valley/minima on the smoothing curves) and peaks (summit/maxima on the smoothing curve) in the smoothed curves were then detected using the "findpeaks" function in R package "pracma".

Consensus-RAM (CRAM) identification. We first identified RAM boundaries in the 93 normal or 19 cancer samples using different step sizes (10kb, 50kb, 100kb, 250kb and $500 \mathrm{~kb}$ ) and then counted the percentage of a genomic region identified as RAM boundary in the 93 normal or 19 cancer samples. A genomic region with occurring percentage $>=25 \%$ was considered as a consensus-RAM (CRAM) boundary in the normal or cancer samples. We merged cRAM boundaries if they are located $<250 \mathrm{~kb}$ apart from each other and required cRAMs have size $>250 \mathrm{~kb}$.

\section{$\mathrm{Hi}-\mathrm{C}$ data analysis}

Hi-C processing. The Hi-C data for the wildtype K562, GM12878, A549, IMR90, NHEK, HUVEC, HMEC and the HCT116 cell lines were downloaded from GEO (GSE63525) and the ENCODE portal (ENCSR662QKG and GSE104333). All the raw fastq files were aligned to hg19 genome and then processed using Juicer with the default settings $(7,49)$. The contact reads in a given cell line were further normalized by vanilla coverage (VC) normalization using the Juicer pipeline. The significance for a given fragment contact was computed by Poisson distribution with VC-normalized expected contact reads versus the VC-normalized observed contact reads. We then used HiCExplorer(50-52) and

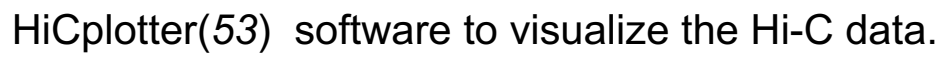

A/B compartment. We performed A/B compartment analysis at $250 \mathrm{~kb}$ resolution. The eigenvectors for each chromosome in all the cell lines involved in the $\mathrm{Hi}-\mathrm{C}$ data analysis were extracted from the $\mathrm{VC}$ normalized $\mathrm{Hi}-\mathrm{C}$ counts processed by the Juicer pipeline with the default parameters(54). POLR2A ChIP-seq data were obtained from the ENCODE(55) portal (https://www.encodeproject.org/). To determine A or B 
compartment, we calculated the correlation between the first eigenvector of each chromosome and the Pol II peak density(56). As there was no Pol II ChIP-seq data available for HMEC, we used TSS density in the hg19 genome to assign A/B compartments in HMEC.

Topological associated domains (TAD). To identify topological associated domains (TAD), we applied the insulation score method(57) to the $\mathrm{Hi}-\mathrm{C}$ data at $50 \mathrm{~kb}$ resolution in the K562, GM12878, A549, IMR90, NHEK, HUVEC, HMEC and HCT116 cell lines. The HFF cell line TADs were downloaded from the 4DN website (accession number 4DNFIMROE6N4). The H1 cell line TADs were downloaded from ref (4).

\section{Lamina-associated domains (LADs) data processing}

The Lamin-B1 signal data in the K562, HCT116, H1, HAP1, RPE-hTERT, HFFc6 cell lines generated by the DamID technique were obtained from the 4DN portal(58). We downloaded the mean of the replicates for each cell line. The Lamin-B1 signals at 50kb resolution for the K562, HCT116, H1, HAP1, RPE-hTERT, HFFc6 cell lines were lifted over from hg38 to hg19.

\section{Cohesin degradation analysis.}

The H3K27ac ChIP-seq data in the untreated HCT-116 RAD21-mAC cells and HCT-116 RAD21-mAC cells treated for 6 hours with IAA were downloaded from GEO (GSE104888). We processed the H3K27ac data same as ref (59). In brief, we aligned the raw data to the hg19 human genome using the BWA software(60), and then deduplicated the reads using PicardTools. The narrow peaks were called by comparing the associated input data using MACS2(61). All the parameters were set to the defaults.

Enrichment of upregulated genes in enhancer-promoter pairs occurring in the same RAM of K562 but split in the background cell type by Hypergeometric Test.

The hypergeometric test was employed to measure the significance of the upregulated genes involved in the enhancer-promoter pairs occurring in the same RAM in K562 (foreground cell type) but in different RAMs of the background cell type. The population size $\mathrm{N}$ was the overall genes involved in the K562 and the compared cell type. The population success size M was the number of all upregulated genes in the K562 compared to the background cell type. The sampling size $\mathrm{n}$ was the number of the genes involved in the enhancer-promoter pairs occurring in the same RAM of K562 but in different RAMs of the background cell type, and the sampling success size $m$ was the upregulated genes involved in the enhancer-promoter pairs occurring in the same RAM of K562 but in different RAMs of the background cell type. Enrichment was considered significant if $p$-value $<0.05$. 
Enrichment of the somatic variants in cancer CRAM boundaries compared to the TAD boundaries assessed by Two-sample Proportion Tests.

The two-sample proportion test null hypothesis was to test the equal proportion of the number of the somatic variants relative to the genome coverage (in base pair) in the cancer CRAM boundaries and TAD boundaries. The two proportions were calculated separately by the number of the somatic variants divided by the boundary length (in base pair) for cancer CRAM boundaries and TAD boundaries. Enrichment was considered significant if $p$-value $<0.05$.

\section{Somatic mutations and structural variation analysis for cancer patients from PCAWG}

The consensus somatic SNV and indels were downloaded from PCAWG. (https://dcc.icgc.org/releases/PCAWG)(62, 63). The VCF files were transformed to bed

files by BEDOPS vcf2bed tools (https://bedops.readthedocs.io/en/latest/content/reference/filemanagement/conversion/vcf2bed.html)(64). The number of somatic SNV and indels overlapping with the RAM and TAD boundaries were then counted.

\section{Motif analysis}

The motif analysis was done using the Homer pipeline (40) with default parameters. The motif occurrence was called using FIMO (41) with $p$-value <=1E-4. 


\section{Figures}

A

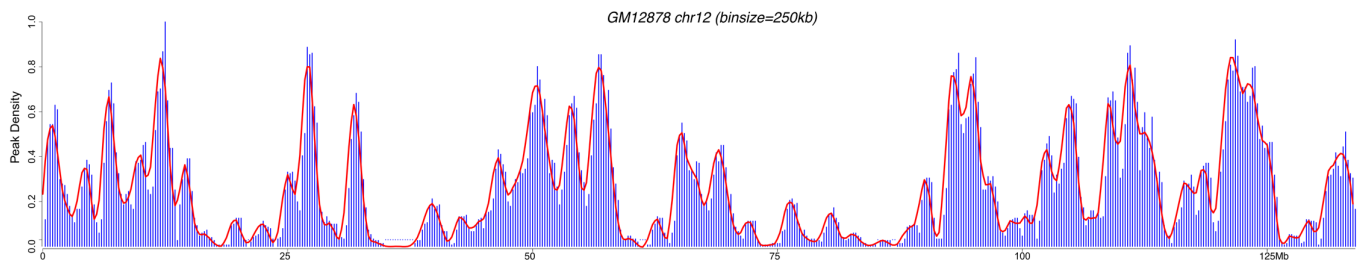

B



D



C

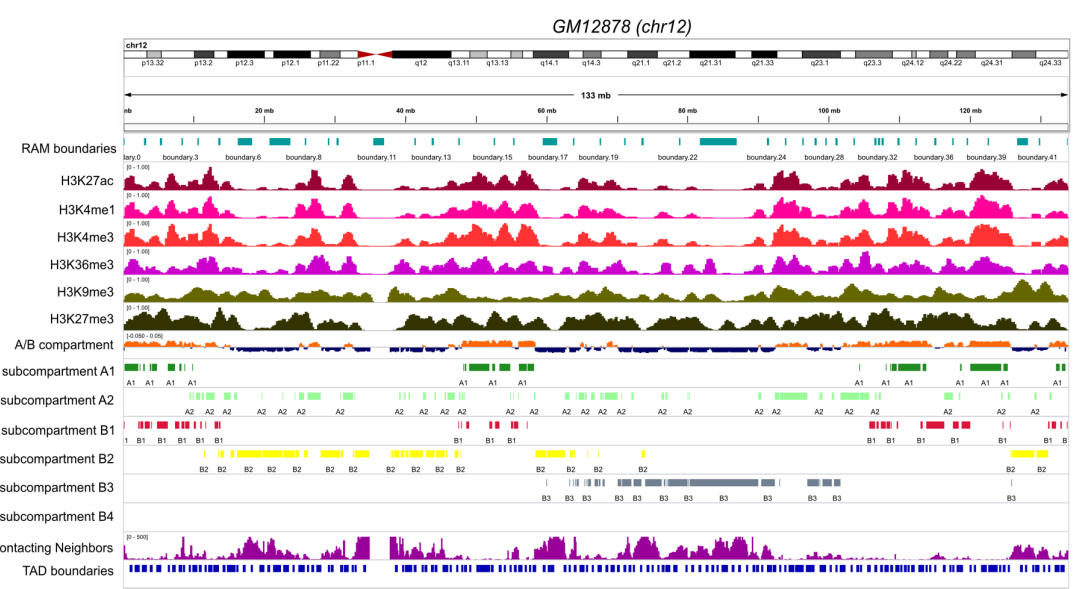

E

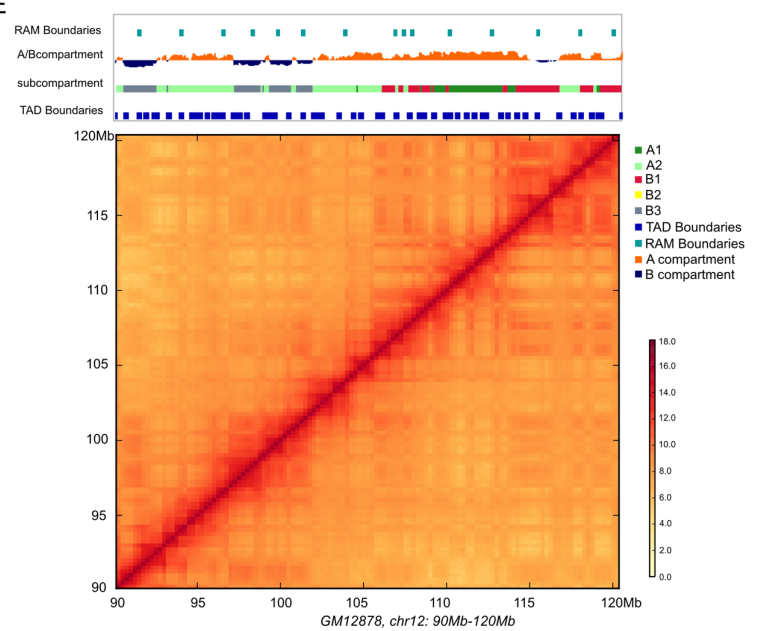

Figure 1. Regulation associated module (RAM) identification. (A) H3K27ac peaks density of chr12 in GM12878 (binsize=250kb) (B) Pearson correlation between histone 
modification marks (H3K27ac, H3K4me1, H3K4me3, H3K36me3, H3K27me3, and H3K9me3) for the Roadmap samples. (C) Examples of histone modifications, A/B compartments, subcompartments, number of the 3D contacts, TAD boundaries and RAM boundaries in chr12 for GM12878. (D) The zoom-in genomic view for chr12:40Mb-80Mb and $(\mathrm{E})$ The zoom-in genomic view for chr12:90Mb-120Mb in hg19. 



H
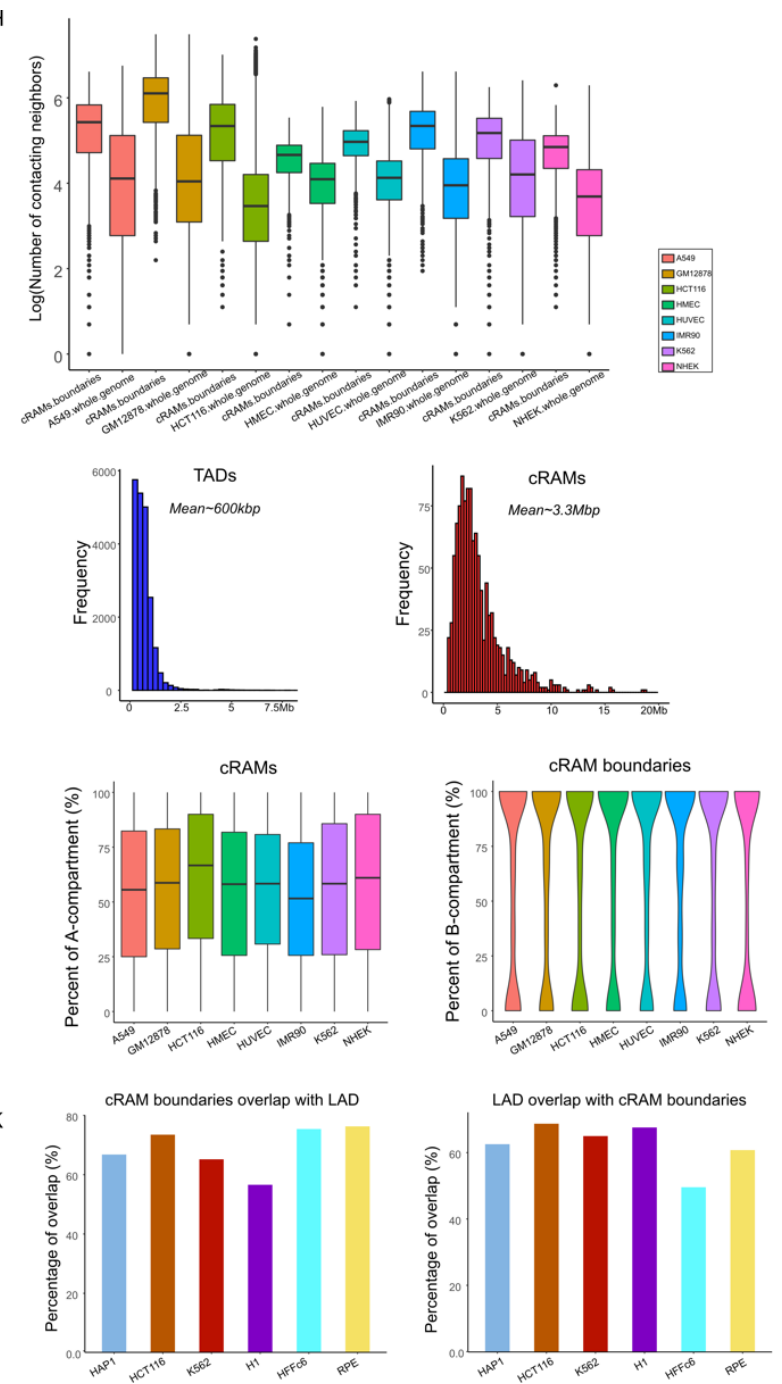

Figure 2. Characterization of the cRAMs and boundaries. (A) Genome browser examples of normal cRAM boundaries and the histone modifications in genomic region chr12:40Mb-90Mb in hg19 for GM12878 and the HUVEC cells. (B-G) The genome-wide enrichment of the histone modification marks. (B) H3K27ac, (C) H3K4me1, (D) H3K4me3, (E) H3K27me3, (F) H3K9me3, and (G) H3K36me3 in cRAM boundaries and non-boundaries $(\mathbf{H})$ The contacting neighbors distribution of cRAM boundaries and whole genome locus in the 3D contact network in a diverse of the cell types. (I) Sizes of the TADs and cRAMs. (J) cRAM boundaries distribution over A/B compartments. (K) cRAM boundaries distribution over LaminB1 signals (LAD). 
A

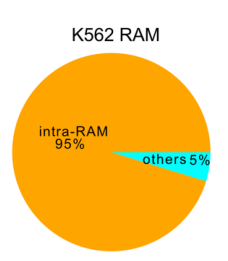

B

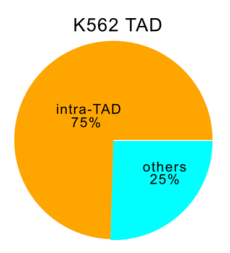

C

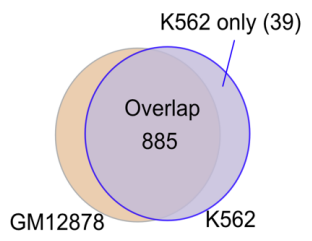

E



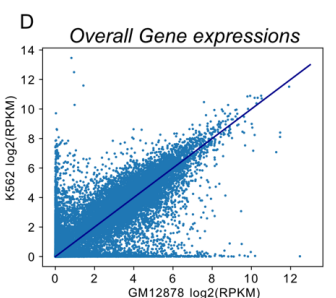

F Overall Gene expressions

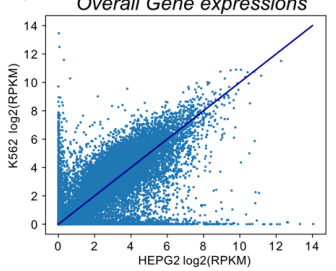

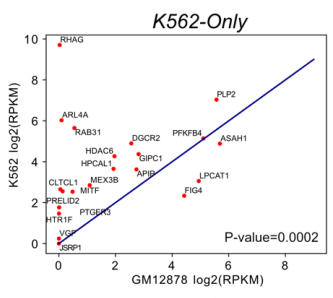

K562-Only

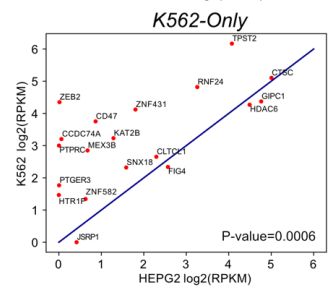

G

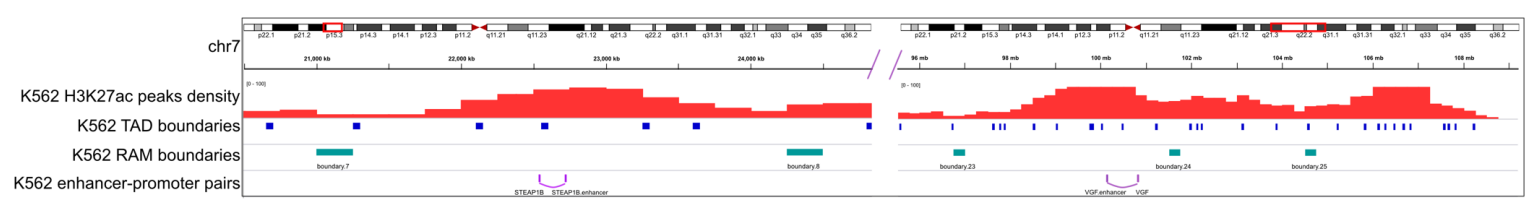

$\mathrm{H}$
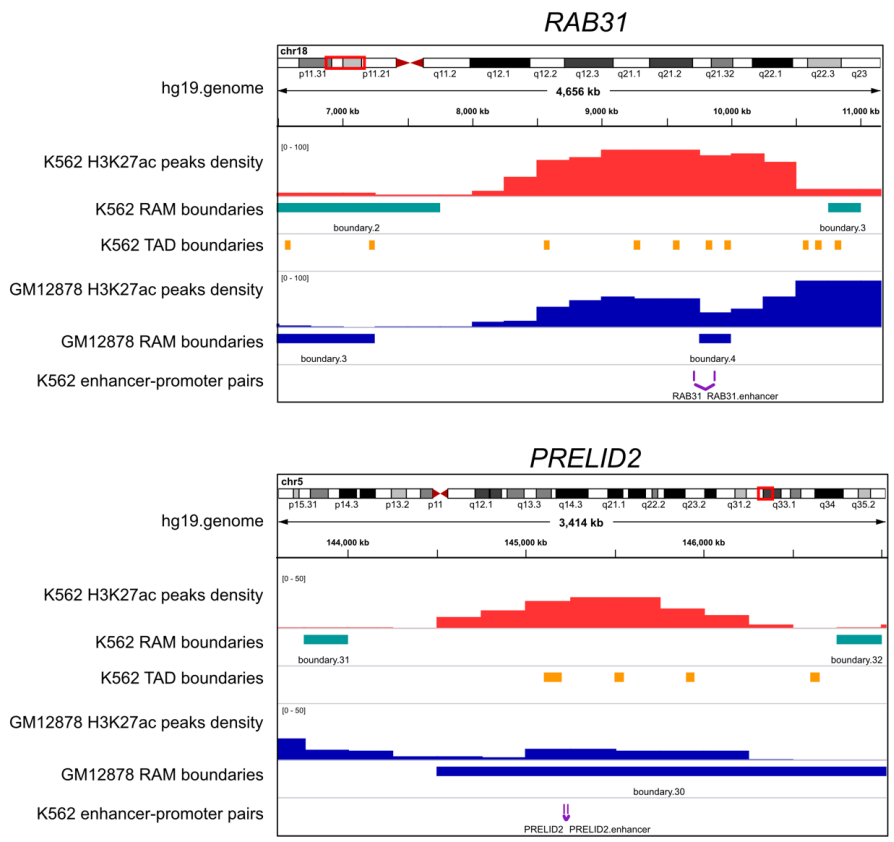

।

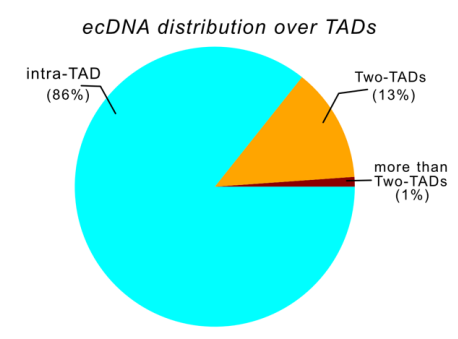

$J$

ecDNA distribution over cancer cRAMs

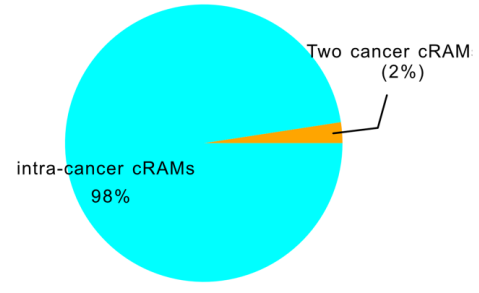

Figure 3 RAM is a functional unit. (A) K562 enhancer-promoter pairs distribution over K562 RAMs. (B) K562 enhancer-promoter pairs distribution over K562 TADs. (C) K562 enhancer-promoter pairs distribution over K562 RAMs and GM12878 RAMs. (D) Genes regulated by the 39 enhancer-promoter interactions only within K562 RAMs tend to have higher expressions in K562 compared to GM12878. (E) K562 enhancer-promoter pairs 
distribution over K562 RAMs and HEPG2 RAMs. (F) Genes regulated by the 28 enhancer-promoter interactions only within K562 RAMs tend to have higher expressions in K562 compared to HEPG2. (G) Examples of K562 enhancer-promoter pairs relative to K562 TAD and RAM boundaries. (H) Examples of K562 enhancer-promoter pairs relative to K562 and GM12878 RAM boundaries. (I) ecDNA distribution over TADs. (J) ecDNA distribution over cancer cRAMs. 

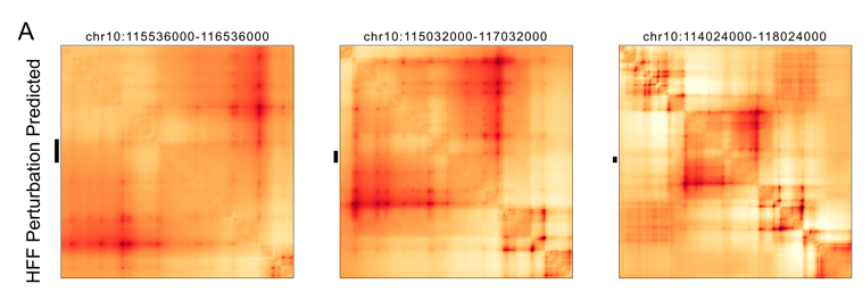

B
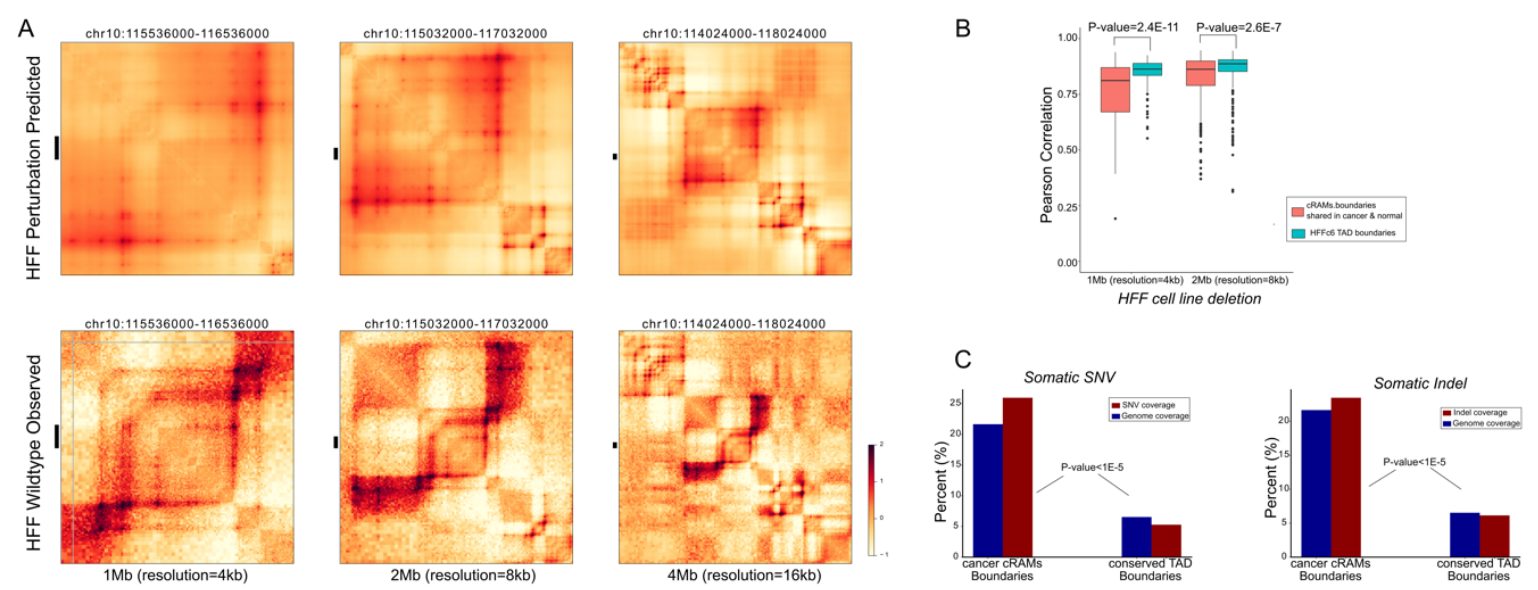

D

\begin{tabular}{|c|c|}
\hline is specific & ndarie \\
\hline Enriched Motif & Best match (P-value) \\
\hline TACTGGCCA & $\begin{array}{l}\text { BCL11A (1E-45) } \\
\text { (crwstogtcaAa) }\end{array}$ \\
\hline ACACTCACTG & $\begin{array}{l}\text { MSANTT3 (1E-26) } \\
\text { (GACACTCAC) }\end{array}$ \\
\hline AGTCCTCT & $\begin{array}{l}\text { SRSF1 (1E-21) } \\
\text { (NTGrocto) }\end{array}$ \\
\hline 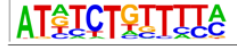 & $\begin{array}{l}\text { STAT2 (1E-20) } \\
\text { (NNTrTCromTr) }\end{array}$ \\
\hline
\end{tabular}

E

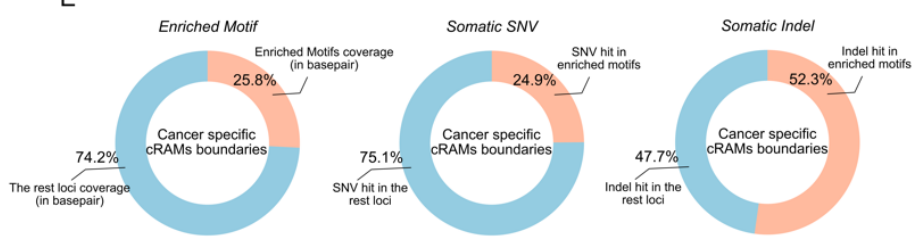

$\mathrm{F}$

\begin{tabular}{|c|c|}
\hline Motif in normal s & \\
\hline Enriched Motif & Best match (P-value) \\
\hline ECAGGAGCAT & $\begin{array}{l}\text { SRSF9 (1E-23) } \\
\text { (AGGAGCA) }\end{array}$ \\
\hline  & $\begin{array}{l}\text { MEIS2 (1E-18) } \\
\text { (NNCCATAATCATNN) }\end{array}$ \\
\hline AATGAATGAA & $\begin{array}{l}\text { ZNF24 (1E-14) } \\
\text { (GATGATGATG) }\end{array}$ \\
\hline IGTITATTGAAG & $\begin{array}{l}\text { FOXE1 (1E-12) } \\
\text { (NTTGTIATIAGGG) }\end{array}$ \\
\hline
\end{tabular}

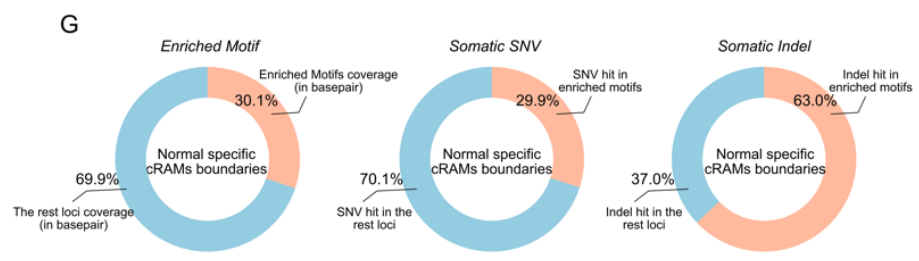

H
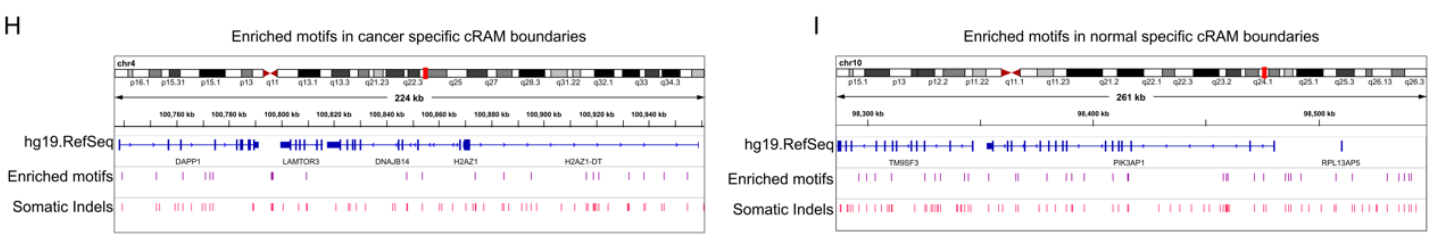

$J$

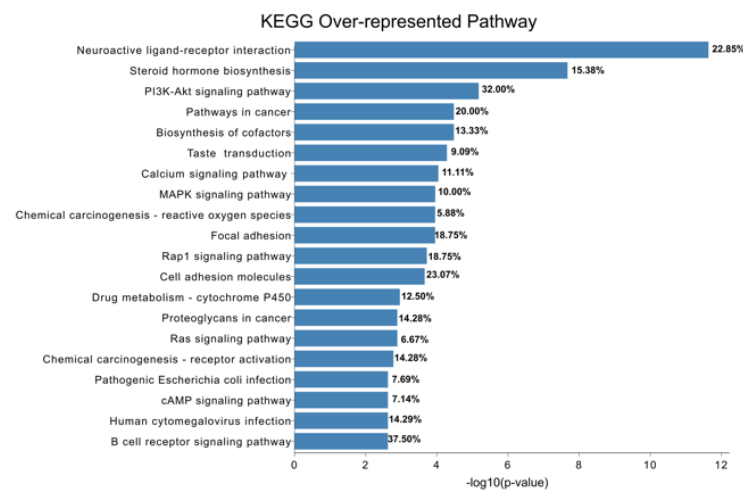

$\mathrm{K}$

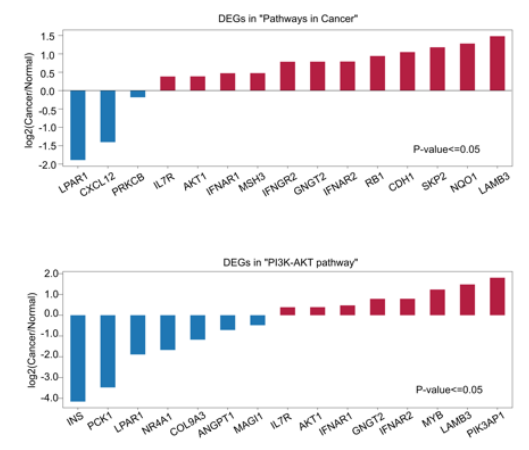

Figure 4. The association of the CRAM boundaries with the 3D chromatin structure and cancer somatic variants. (A) An example of the $\mathrm{Hi}-\mathrm{C}$ contact change upon deletion 
of the cRAM boundary (chr10:115,940,000-116,040,000 in hg38) in HFF cells predicted by a deep learning model ORCA. (B) Pearson correlations between the predicted $\mathrm{Hi}-\mathrm{C}$ contacts before and after CRAM boundary and TAD boundary deletion in HFF cells. A lower correlation indicates a larger perturbation to the wildtype chromatin structure upon deletion. (C) Somatic SNV and indels enrichment in cancer CRAM boundaries and TAD boundaries. Genome coverage: the total base pairs of the cancer CRAM boundaries or TAD boundaries in the whole genome; SNV coverage: the percentage of the SNVs in the cancer CRAM boundaries or TAD boundaries in the whole genome; INDEL coverage: the percentage of the indels in cancer CRAM boundaries or TAD boundaries in the whole genome. (D) Examples of the enriched motifs in the cancer specific CRAM boundaries. (E) Overlaps of somatic SNVs and indels with the enriched motifs in the cancer specific cRAM boundaries (F) Examples of the enriched motifs in the normal specific cRAM boundaries. (G) Overlaps of somatic SNVs and indels with the enriched motifs in the normal specific cRAM boundaries $(\mathbf{H})$ The zoom-in genomic view for the enriched motifs with somatic indels in cancer specific cRAM boundaries (I) The zoom-in genomic view for the enriched motifs with somatic indels in normal specific cRAM boundaries (J) KEGG over-represented pathways for the genes within $2.5 \mathrm{~kb}$ from the enriched motifs overlapping with somatic indels (K) Differentially expressed genes in KEGG "Pathways in cancer" and "PI3K-AKT pathway" 


\section{REFERENCES}

1. T. Kouzarides, Chromatin modifications and their function. Cell. 128, 693-705 (2007).

2. B. Li, M. Carey, J. L. Workman, The role of chromatin during transcription. Cell. 128, 707-719 (2007).

3. E. P. Nora, B. R. Lajoie, E. G. Schulz, L. Giorgetti, I. Okamoto, N. Servant, T. Piolot, N. L. van Berkum, J. Meisig, J. Sedat, J. Gribnau, E. Barillot, N. Blüthgen, J. Dekker, E. Heard, Spatial partitioning of the regulatory landscape of the Xinactivation centre. Nature. 485 (2012), pp. 381-385.

4. J. R. Dixon, S. Selvaraj, F. Yue, A. Kim, Y. Li, Y. Shen, M. Hu, J. S. Liu, B. Ren, Topological domains in mammalian genomes identified by analysis of chromatin interactions. Nature. 485, 376-380 (2012).

5. T. Sexton, E. Yaffe, E. Kenigsberg, F. Bantignies, B. Leblanc, M. Hoichman, H. Parrinello, A. Tanay, G. Cavalli, Three-dimensional folding and functional organization principles of the Drosophila genome. Cell. 148, 458-472 (2012).

6. E. Crane, Q. Bian, R. P. McCord, B. R. Lajoie, B. S. Wheeler, E. J. Ralston, S. Uzawa, J. Dekker, B. J. Meyer, Condensin-driven remodelling of X chromosome topology during dosage compensation. Nature. 523 (2015), pp. 240-244.

7. S. S. P. Rao, M. H. Huntley, N. C. Durand, E. K. Stamenova, I. D. Bochkov, J. T. Robinson, A. L. Sanborn, I. Machol, A. D. Omer, E. S. Lander, E. L. Aiden, A 3D map of the human genome at kilobase resolution reveals principles of chromatin looping. Cell. 159, 1665-1680 (2014).

8. E. Lieberman-Aiden, N. L. van Berkum, L. Williams, M. Imakaev, T. Ragoczy, A. Telling, I. Amit, B. R. Lajoie, P. J. Sabo, M. O. Dorschner, R. Sandstrom, B. Bernstein, M. A. Bender, M. Groudine, A. Gnirke, J. Stamatoyannopoulos, L. A. Mirny, E. S. Lander, J. Dekker, Comprehensive Mapping of Long-Range Interactions Reveals Folding Principles of the Human Genome. Science. 326 (2009), pp. 289-293.

9. Y. Zhu, Z. Chen, K. Zhang, M. Wang, D. Medovoy, J. W. Whitaker, B. Ding, N. Li, L. Zheng, W. Wang, Constructing 3D interaction maps from 1D epigenomes. Nat. Commun. 7, 10812 (2016).

10. J. Huang, E. Marco, L. Pinello, G.-C. Yuan, Predicting chromatin organization using histone marks. Genome Biol. 16, 162 (2015).

11. H. Ashoor, X. Chen, W. Rosikiewicz, J. Wang, A. Cheng, P. Wang, Y. Ruan, S. Li, Graph embedding and unsupervised learning predict genomic sub-compartments from HiC chromatin interaction data. Nat. Commun. 11, 1173 (2020). 
12. A. J. Bannister, T. Kouzarides, Regulation of chromatin by histone modifications. Cell Res. 21, 381-395 (2011).

13. M. Vermeulen, H. C. Eberl, F. Matarese, H. Marks, S. Denissov, F. Butter, K. K. Lee, J. V. Olsen, A. A. Hyman, H. G. Stunnenberg, M. Mann, Quantitative interaction proteomics and genome-wide profiling of epigenetic histone marks and their readers. Cell. 142, 967-980 (2010).

14. T. Bartke, M. Vermeulen, B. Xhemalce, S. C. Robson, M. Mann, T. Kouzarides, Nucleosome-interacting proteins regulated by DNA and histone methylation. Cell. 143, 470-484 (2010).

15. Z. Liu, W. R. Legant, B.-C. Chen, L. Li, J. B. Grimm, L. D. Lavis, E. Betzig, R. Tjian, 3D imaging of Sox2 enhancer clusters in embryonic stem cells. Elife. 3, e04236 (2014).

16. I. I. Cisse, I. Izeddin, S. Z. Causse, L. Boudarene, A. Senecal, L. Muresan, C. Dugast-Darzacq, B. Hajj, M. Dahan, X. Darzacq, Real-time dynamics of RNA polymerase II clustering in live human cells. Science. 341, 664-667 (2013).

17. W.-K. Cho, J.-H. Spille, M. Hecht, C. Lee, C. Li, V. Grube, I. I. Cisse, Mediator and RNA polymerase II clusters associate in transcription-dependent condensates. Science. 361, 412-415 (2018).

18. A. Pancholi, T. Klingberg, W. Zhang, R. Prizak, I. Mamontova, A. Noa, M. Sobucki, A. Y. Kobitski, G. U. Nienhaus, V. Zaburdaev, L. Hilbert, RNA polymerase II clusters form in line with surface condensation on regulatory chromatin. Mol. Syst. Biol. 17, e10272 (2021).

19. Roadmap Epigenomics Consortium, A. Kundaje, W. Meuleman, J. Ernst, M. Bilenky, A. Yen, A. Heravi-Moussavi, P. Kheradpour, Z. Zhang, J. Wang, M. J. Ziller, V. Amin, J. W. Whitaker, M. D. Schultz, L. D. Ward, A. Sarkar, G. Quon, R. S. Sandstrom, M. L. Eaton, Y.-C. Wu, A. R. Pfenning, X. Wang, M. Claussnitzer, Y. Liu, C. Coarfa, R. A. Harris, N. Shoresh, C. B. Epstein, E. Gjoneska, D. Leung, W. Xie, R. D. Hawkins, R. Lister, C. Hong, P. Gascard, A. J. Mungall, R. Moore, E. Chuah, A. Tam, T. K. Canfield, R. S. Hansen, R. Kaul, P. J. Sabo, M. S. Bansal, A. Carles, J. R. Dixon, K.-H. Farh, S. Feizi, R. Karlic, A.-R. Kim, A. Kulkarni, D. Li, R. Lowdon, G. Elliott, T. R. Mercer, S. J. Neph, V. Onuchic, P. Polak, N. Rajagopal, P. Ray, R. C. Sallari, K. T. Siebenthall, N. A. Sinnott-Armstrong, M. Stevens, R. E. Thurman, J. Wu, B. Zhang, X. Zhou, A. E. Beaudet, L. A. Boyer, P. L. De Jager, P. J. Farnham, S. J. Fisher, D. Haussler, S. J. M. Jones, W. Li, M. A. Marra, M. T. McManus, S. Sunyaev, J. A. Thomson, T. D. Tlsty, L.-H. Tsai, W. Wang, R. A. Waterland, M. Q. Zhang, L. H. Chadwick, B. E. Bernstein, J. F. Costello, J. R. Ecker, M. Hirst, A. Meissner, A. Milosavljevic, B. Ren, J. A. Stamatoyannopoulos, T. Wang, M. Kellis, Integrative analysis of 111 reference human epigenomes. Nature. 518, 317-330 (2015).

20. C. A. Sloan, E. T. Chan, J. M. Davidson, V. S. Malladi, J. S. Strattan, B. C. Hitz, I. 
Gabdank, A. K. Narayanan, M. Ho, B. T. Lee, L. D. Rowe, T. R. Dreszer, G. Roe, N. R. Podduturi, F. Tanaka, E. L. Hong, J. M. Cherry, ENCODE data at the ENCODE portal. Nucleic Acids Res. 44, D726-32 (2016).

21. J. T. Robinson, H. Thorvaldsdóttir, W. Winckler, M. Guttman, E. S. Lander, G. Getz, J. P. Mesirov, Integrative genomics viewer. Nat. Biotechnol. 29, 24-26 (2011).

22. R. Foisner, L. Gerace, Integral membrane proteins of the nuclear envelope interact with lamins and chromosomes, and binding is modulated by mitotic phosphorylation. Cell. 73, 1267-1279 (1993).

23. K. L. Wydner, J. A. McNeil, F. Lin, H. J. Worman, J. B. Lawrence, Chromosomal assignment of human nuclear envelope protein genes LMNA, LMNB1, and LBR by fluorescence in situ hybridization. Genomics. 32, 474-478 (1996).

24. M. Gasperini, A. J. Hill, J. L. McFaline-Figueroa, B. Martin, S. Kim, M. D. Zhang, D. Jackson, A. Leith, J. Schreiber, W. S. Noble, C. Trapnell, N. Ahituv, J. Shendure, A Genome-wide Framework for Mapping Gene Regulation via Cellular Genetic Screens. Cell. 176, 1516 (2019).

25. C. P. Fulco, J. Nasser, T. R. Jones, G. Munson, D. T. Bergman, V. Subramanian, S. R. Grossman, R. Anyoha, B. R. Doughty, T. A. Patwardhan, T. H. Nguyen, M. Kane, E. M. Perez, N. C. Durand, C. A. Lareau, E. K. Stamenova, E. L. Aiden, E. S. Lander, J. M. Engreitz, Activity-by-contact model of enhancer-promoter regulation from thousands of CRISPR perturbations. Nature Genetics. 51 (2019), pp. 16641669.

26. A.-L. Valton, J. Dekker, TAD disruption as oncogenic driver. Curr. Opin. Genet. Dev. 36, 34-40 (2016).

27. A. D. Rouillard, G. W. Gundersen, N. F. Fernandez, Z. Wang, C. D. Monteiro, M. G. McDermott, A. Ma'ayan, The harmonizome: a collection of processed datasets gathered to serve and mine knowledge about genes and proteins. Database . 2016 (2016), doi:10.1093/database/baw100.

28. M. Cremer, K. Brandstetter, A. Maiser, S. S. P. Rao, V. J. Schmid, M. Guirao-Ortiz, N. Mitra, S. Mamberti, K. N. Klein, D. M. Gilbert, H. Leonhardt, M. C. Cardoso, E. L. Aiden, H. Harz, T. Cremer, Cohesin depleted cells rebuild functional nuclear compartments after endomitosis. Nat. Commun. 11, 6146 (2020).

29. S. S. P. Rao, S.-C. Huang, B. Glenn St Hilaire, J. M. Engreitz, E. M. Perez, K.-R. Kieffer-Kwon, A. L. Sanborn, S. E. Johnstone, G. D. Bascom, I. D. Bochkov, X. Huang, M. S. Shamim, J. Shin, D. Turner, Z. Ye, A. D. Omer, J. T. Robinson, T. Schlick, B. E. Bernstein, R. Casellas, E. S. Lander, E. L. Aiden, Cohesin Loss Eliminates All Loop Domains. Cell. 171, 305-320.e24 (2017).

30. K. M. Turner, V. Deshpande, D. Beyter, T. Koga, J. Rusert, C. Lee, B. Li, K. Arden, B. Ren, D. A. Nathanson, H. I. Kornblum, M. D. Taylor, S. Kaushal, W. K. Cavenee, 
R. Wechsler-Reya, F. B. Furnari, S. R. Vandenberg, P. N. Rao, G. M. Wahl, V. Bafna, P. S. Mischel, Extrachromosomal oncogene amplification drives tumour evolution and genetic heterogeneity. Nature. 543, 122-125 (2017).

31. R. G. W. Verhaak, V. Bafna, P. S. Mischel, Extrachromosomal oncogene amplification in tumour pathogenesis and evolution. Nat. Rev. Cancer. 19, 283-288 (2019).

32. A. C. deCarvalho, H. Kim, L. M. Poisson, M. E. Winn, C. Mueller, D. Cherba, J. Koeman, S. Seth, A. Protopopov, M. Felicella, S. Zheng, A. Multani, Y. Jiang, J. Zhang, D.-H. Nam, E. F. Petricoin, L. Chin, T. Mikkelsen, R. G. W. Verhaak, Discordant inheritance of chromosomal and extrachromosomal DNA elements contributes to dynamic disease evolution in glioblastoma. Nat. Genet. 50, 708-717 (2018).

33. H. Kim, N.-P. Nguyen, K. Turner, S. Wu, A. D. Gujar, J. Luebeck, J. Liu, V. Deshpande, U. Rajkumar, S. Namburi, S. B. Amin, E. Yi, F. Menghi, J. H. Schulte, A. G. Henssen, H. Y. Chang, C. R. Beck, P. S. Mischel, V. Bafna, R. G. W. Verhaak, Extrachromosomal DNA is associated with oncogene amplification and poor outcome across multiple cancers. Nat. Genet. 52, 891-897 (2020).

34. C. Y. McLean, D. Bristor, M. Hiller, S. L. Clarke, B. T. Schaar, C. B. Lowe, A. M. Wenger, G. Bejerano, GREAT improves functional interpretation of cis-regulatory regions. Nat. Biotechnol. 28, 495-501 (2010).

35. S. Sigismund, D. Avanzato, L. Lanzetti, Emerging functions of the EGFR in cancer. Mol. Oncol. 12, 3-20 (2018).

36. I. S. Babina, N. C. Turner, Advances and challenges in targeting FGFR signalling in cancer. Nat. Rev. Cancer. 17, 318-332 (2017).

37. M. Sahraei, L. D. Roy, J. M. Curry, T. L. Teresa, S. Nath, D. Besmer, A. Kidiyoor, R. Dalia, S. J. Gendler, P. Mukherjee, MUC1 regulates PDGFA expression during pancreatic cancer progression. Oncogene. 31, 4935-4945 (2012).

38. J. Zhou, Sequence-based modeling of genome 3D architecture from kilobase to chromosome-scale. bioRxiv (2021), p. 2021.05.19.444847.

39. T. I. P.-C. A. of W. G. Consortium, The ICGC/TCGA Pan-Cancer Analysis of Whole Genomes Consortium, Pan-cancer analysis of whole genomes. Nature. 578 (2020), pp. 82-93.

40. S. Heinz, C. Benner, N. Spann, E. Bertolino, Y. C. Lin, P. Laslo, J. X. Cheng, C. Murre, H. Singh, C. K. Glass, Simple Combinations of Lineage-Determining Transcription Factors Prime cis-Regulatory Elements Required for Macrophage and B Cell Identities. Molecular Cell. 38 (2010), pp. 576-589.

41. C. E. Grant, T. L. Bailey, W. S. Noble, FIMO: scanning for occurrences of a given 
motif. Bioinformatics. 27, 1017-1018 (2011).

42. U. Raudvere, L. Kolberg, I. Kuzmin, T. Arak, P. Adler, H. Peterson, J. Vilo, $\mathrm{g}$ :Profiler: a web server for functional enrichment analysis and conversions of gene lists (2019 update). Nucleic Acids Res. 47, W191-W198 (2019).

43. V. A. Bloomfield, Condensation of DNA by multivalent cations: considerations on mechanism. Biopolymers. 31, 1471-1481 (1991).

44. I. Koltover, K. Wagner, C. R. Safinya, DNA condensation in two dimensions. Proc. Natl. Acad. Sci. U. S. A. 97, 14046-14051 (2000).

45. A. Srivastava, R. Timsina, S. Heo, S. W. Dewage, S. Kirmizialtin, X. Qiu, Structureguided DNA-DNA attraction mediated by divalent cations. Nucleic Acids Res. 48, 7018-7026 (2020).

46. C. A. Davey, T. J. Richmond, DNA-dependent divalent cation binding in the nucleosome core particle. Proc. Natl. Acad. Sci. U. S. A. 99, 11169-11174 (2002).

47. Z. Yang, J. J. Hayes, The divalent cations Ca2+ and Mg2+ play specific roles in stabilizing histone-DNA interactions within nucleosomes that are partially redundant with the core histone tail domains. Biochemistry. 50, 9973-9981 (2011).

48. W. S. Cleveland, E. Grosse, Computational methods for local regression. Statistics and Computing. 1 (1991), pp. 47-62.

49. N. C. Durand, M. S. Shamim, I. Machol, S. S. P. Rao, M. H. Huntley, E. S. Lander, E. L. Aiden, Juicer Provides a One-Click System for Analyzing Loop-Resolution HiC Experiments. Cell Syst. 3, 95-98 (2016).

50. J. Wolff, L. Rabbani, R. Gilsbach, G. Richard, T. Manke, R. Backofen, B. A. Grüning, Galaxy HiCExplorer 3: a web server for reproducible $\mathrm{Hi}-\mathrm{C}$, capture $\mathrm{Hi}-\mathrm{C}$ and single-cell Hi-C data analysis, quality control and visualization. Nucleic Acids Res. 48, W177-W184 (2020).

51. F. Ramírez, V. Bhardwaj, L. Arrigoni, K. C. Lam, B. A. Grüning, J. Villaveces, B. Habermann, A. Akhtar, T. Manke, High-resolution TADs reveal DNA sequences underlying genome organization in flies. Nat. Commun. 9, 189 (2018).

52. J. Wolff, V. Bhardwaj, S. Nothjunge, G. Richard, G. Renschler, R. Gilsbach, T. Manke, R. Backofen, F. Ramírez, B. A. Grüning, Galaxy HiCExplorer: a web server for reproducible Hi-C data analysis, quality control and visualization. Nucleic Acids Res. 46, W11-W16 (2018).

53. K. C. Akdemir, L. Chin, HiCPlotter integrates genomic data with interaction matrices. Genome Biol. 16, 198 (2015).

54. N. C. Durand, M. S. Shamim, I. Machol, S. S. P. Rao, M. H. Huntley, E. S. Lander, 
E. L. Aiden, Juicer Provides a One-Click System for Analyzing Loop-Resolution HiC Experiments. Cell Syst. 3, 95-98 (2016).

55. K. Y. Yip, C. Cheng, N. Bhardwaj, J. B. Brown, J. Leng, A. Kundaje, J. Rozowsky, E. Birney, P. Bickel, M. Snyder, M. Gerstein, Classification of human genomic regions based on experimentally determined binding sites of more than 100 transcription-related factors. Genome Biol. 13, R48 (2012).

56. R. Kalhor, H. Tjong, N. Jayathilaka, F. Alber, L. Chen, Genome architectures revealed by tethered chromosome conformation capture and population-based modeling. Nat. Biotechnol. 30, 90-98 (2011).

57. E. Crane, Q. Bian, R. P. McCord, B. R. Lajoie, B. S. Wheeler, E. J. Ralston, S. Uzawa, J. Dekker, B. J. Meyer, Condensin-Driven Remodeling of X-Chromosome Topology during Dosage Compensation. Nature. 523, 240 (2015).

58. T. van Schaik, M. Vos, D. Peric-Hupkes, P. Hn Celie, B. van Steensel, Cell cycle dynamics of lamina-associated DNA. EMBO Rep. 21, e50636 (2020).

59. S. S. P. Rao, S.-C. Huang, B. G. St Hilair, J. M. Engreitz, E. M. Perez, K.-R. KiefferKwon, A. L. Sanborn, S. E. Johnstone, I. D. Bochkov, X. Huang, M. S. Shamim, A. D. Omer, B. E. Bernstein, R. Casellas, E. S. Lander, E. L. Aiden, Cohesin loss eliminates all loop domains, leading to links among superenhancers and downregulation of nearby genes, , doi:10.1101/139782.

60. H. Li, R. Durbin, Fast and accurate long-read alignment with Burrows-Wheeler transform. Bioinformatics. 26, 589-595 (2010).

61. T. Liu, Use model-based Analysis of ChIP-Seq (MACS) to analyze short reads generated by sequencing protein-DNA interactions in embryonic stem cells. Methods Mol. Biol. 1150, 81-95 (2014).

62. Pan-cancer analysis of whole genomes. Nature. 578, 82-93 (2020).

63. W. Jiao, G. Atwal, P. Polak, R. Karlic, E. Cuppen, PCAWG Tumor Subtypes and Clinical Translation Working Group, A. Danyi, J. de Ridder, C. van Herpen, M. P. Lolkema, N. Steeghs, G. Getz, Q. Morris, L. D. Stein, PCAWG Consortium, A deep learning system accurately classifies primary and metastatic cancers using passenger mutation patterns. Nat. Commun. 11, 728 (2020).

64. S. Neph, M. S. Kuehn, A. P. Reynolds, E. Haugen, R. E. Thurman, A. K. Johnson, E. Rynes, M. T. Maurano, J. Vierstra, S. Thomas, R. Sandstrom, R. Humbert, J. A. Stamatoyannopoulos, BEDOPS: high-performance genomic feature operations. Bioinformatics. 28, 1919-1920 (2012). 